\title{
Uptake of genetic counselling and predictive DNA testing in hypertrophic cardiomyopathy
}

\author{
Imke Christiaans ${ }^{*, 1}$, Erwin Birnie ${ }^{2}$, Gouke J Bonsel ${ }^{2}$, Arthur AM Wilde ${ }^{3}$ and \\ Irene $\mathrm{M}$ van Langen ${ }^{1}$
}

${ }^{1}$ Department of Clinical Genetics, Academic Medical Centre, Amsterdam, The Netherlands; ${ }^{2}$ Institute of Health Policy
and Management, Erasmus Medical Centre, Rotterdam, the Netherlands; ${ }^{3}$ Department of Cardiology, Academic
Medical Centre, Amsterdam, The Netherlands

Hypertrophic cardiomyopathy is a common autosomal dominant disease, associated with heart failure and arrhythmias predisposing to sudden cardiac death. After the detection of the causal mutation in the proband predictive DNA testing of relatives is possible (cascade screening). Prevention of sudden cardiac death in patients with a high risk by means of an implantable cardioverter defibrillator is effective. In 97 hypertrophic cardiomyopathy families with a sarcomere gene mutation we retrospectively determined uptake of genetic counselling and predictive DNA testing in relatives within 1 year after the detection of the causal mutation in the proband. Uptake of genetic counselling was $39 \%$ and did not differ significantly by proband's or relative's gender, nor by young age of the relative ( $<18$ years) or a family history positive for sudden cardiac death. In second-degree relatives, eligible for predictive DNA testing when the firstdegree relative had died, uptake was $27.5 \%(P=0.047)$. Uptake of predictive genetic testing was $39 \%$; conditional uptake of predictive genetic testing was $99 \%$. Uptake of genetic counselling in hypertrophic cardiomyopathy is comparable to uptake in oncogenetics. Conditional uptake of predictive DNA testing, however, is much higher. Because sudden cardiac death can be prevented uptake of genetic counselling in hypertrophic cardiomyopathy should be as high as possible. To achieve this research into the determinants of uptake is needed.

European Journal of Human Genetics (2008) 16, 1201-1207; doi:10.1038/ejhg.2008.92; published online 14 May 2008

Keywords: hypertrophic cardiomyopathy; genetic counselling; genetic testing; uptake

Introduction

Hypertrophic cardiomyopathy (HCM) is a relatively common genetic disease, affecting about 1 in 500 persons and characterized by asymmetric left ventricular hypertrophy. ${ }^{1-3}$ Many patients are asymptomatic initially and some of them are diagnosed in adult life through routine screening. However, HCM can also be a very

*Correspondence: Dr I Christiaans, Department of Clinical Genetics, Academic Medical Centre, Meibergdreef 15, Amsterdam 1105 AZ, The Netherlands. Tel: + 31205665 111; Fax: + 31206918 626;

E-mail: i.christiaans@amc.uva.nl

Received 22 November 2007; revised 29 February 2008; accepted 9 April 2008; published online 14 May 2008 disabling and potentially lethal disease giving rise to dyspnoea, exertional angina, palpitations, (pre)syncope and sudden death at young age often without prior warning. ${ }^{4}$ Annual mortality rate from sudden cardiac death (SCD) is $1-5 \% .^{5,6}$ In the general population aged 20-75 years annual mortality from SCD was $0.1 \%$ in the 1990s in the Netherlands. ${ }^{7}$

HCM is inherited as an autosomal dominant trait. Mutations in 11 genes, mainly coding for sarcomeric proteins, are now known to predispose for HCM. In more than half of the HCM patients the disease-causing mutation is currently detected. ${ }^{8,9}$ In the Netherlands $90 \%$ of mutations are identified in the myosin-binding protein $\mathrm{C}$ 
(MYBPC3) gene, in about 35-40\% of families this concerns the c.2373_2374insG mutation, one of the three Dutch founder mutations. ${ }^{10}$

Mean age of diagnosis of HCM patients reported in literature is in their late thirties. ${ }^{11-13}$ Genetic DNA testing in HCM patients and their relatives, however, has taught us that disease penetrance is incomplete and mutation carriers can be diagnosed with HCM even in their sixties. ${ }^{14}$ There is no difference in disease penetrance between carriers of the Dutch founder mutation and carriers of other MYBPC3 mutations (I Christiaans, unpublished data).

Predictive genetic testing in relatives of HCM patients can be performed in two ways; predictive cardiological evaluation (electrocardiography (ECG) and echocardiography) or predictive molecular testing (DNA testing). The latter is only possible after the identification of a diseasecausing mutation in an HCM patient and is also known as cascade screening. When the disease-causing mutation is unknown, only cardiological evaluation of relatives is possible. In this way relatives showing the phenotype will be identified, but relatives in whom the disease is not (yet) penetrant will be missed, implying that cardiological evaluation has to be repeated regularly and exclusion from risk of the disease in offspring is not possible. ${ }^{15}$ Although there is as yet no treatment that affects the progression of the disease importantly and it is unknown if detection of the disease in an early stage affects prognosis, primary and secondary prevention in patients with a high risk of SCD by means of an implantable cardioverter defibrillator is effective and individuals at risk should be identified. $^{16,17}$

In families with a disease-causing mutation predictive DNA testing with subsequent cardiological evaluation of mutation carriers has two main advantages. From a public health point of view, it is important to identify as many mutation carriers as possible, including individuals who may be at high risk for sudden death. ${ }^{18}$ These individuals are eligible for preventive strategies if they appear to be at high risk whereas non-carriers can be discharged from further cardiological evaluations. In addition, children with the familial mutation who aim at a professional career in sports can be advised against this. From a genetic counselling point of view, it is important to identify and inform relatives about the value of predictive DNA testing. For this reason we have chosen to systematically and proactively offer genetic counselling and predictive DNA testing to all eligible relatives in families with a diseasecausing mutation. ${ }^{19}$

The aims of prevention and counselling can only be achieved if both the uptake of genetic counselling and predictive DNA testing are optimal. As part of an evaluation of our current strategy we assessed the uptake of genetic counselling and predictive DNA testing in our population of HCM families with a disease-causing mutation.

\section{Methods \\ Setting}

In our multidisciplinary cardiogenetics outpatient clinic genetic counselling and cascade screening for HCM starts with a proband, the HCM patient. Counselling sessions, in probands and relatives, always combine the consultation of a cardiologist and a clinical geneticist (or genetic counsellor). Support from a psychosocial worker is actively offered. After the detection of a pathogenic mutation we ask the proband to inform the first-degree relatives - or seconddegree in case the first-degree relative is deceased - by means of a family letter, about the aims, opportunities and possible drawbacks of predictive genetic testing. ${ }^{20}$ If necessary, psychosocial support and suggestions for strategies to approach the family are actively offered. In the family letter relatives are also referred to our website, where more information about the disease and predictive DNA testing can be found (www.cardiogenetica.nl) and all close relatives are invited for referral to our clinic for genetic counselling, including discussion of the pros and cons of DNA testing. We use adapted Huntington guidelines for predictive testing in heritable heart diseases, like HCM (Table 1). ${ }^{19}$ In a counselling session for predictive DNA testing in our cardiogenetics outpatient clinic the relative is informed about the disease, the risk of SCD and the pros and cons of predictive cardiological and DNA testing. An ECG is made before the session that can, after counselling, be interpreted by the attending cardiologist as a predictive clinical test (with low sensitivity and specificity however) on demand of the counselee. Before DNA testing psychosocial support is offered actively. A session with a social worker can take place directly after the counselling session or at a second appointment or by telephone. Psychosocial support is not obligatory before genetic DNA testing, except when minors are tested. DNA testing can, on demand of the counselee, take place directly after the first session, but if the relative needs more time to consider testing, this is also possible after a second appointment. In our experience most counselees already decided in favour of predictive testing before attending, on the basis of the information supplied before the visit. The test result is given personally at the cardiogenetics outpatient clinic, by telephone or by mail depending on the preference of the relative. Follow-up appointments including psychosocial care are offered to mutation carriers, especially to those having children Carriers of the familial mutation are referred for subsequent cardiological evaluation and regular follow-up aiming at reduction of SCD.

The process from the detection of a disease-causing mutation in a proband towards predictive DNA testing of the proband's relatives consists of five different phases (Figure 1). The first phase (I) concerns informing the proband about the possibility of predictive DNA testing in his or her relatives. In the second phase (II) the relatives should be made acquainted with this information. 
Table 1 Protocol used for genetic DNA-based cascade screening in HCM

1. Genetic counselling (including the drawing of an extended pedigree), extension of cardiological evaluation (if necessary) and genetic testing of the proband in a multidisciplinary cardiogenetics outpatient clinic

2. If a mutation is detected: education of the proband and initiation of cascade screening

3. Informing the first- and second-degree relatives by the proband (if necessary by the medical specialists), using an information letter written by the medical team

4. Genetic counselling of relatives before genetic testing, during a family meeting and/or individually

5. Clinical testing of relatives at first consultation (ECG)

6. Genetic testing of relatives at first consultation (or at a second appointment, if desired by an individual needing more time to consider testing)

7. Psychosocial care (psychologist, social worker) mandatory for all families in whom minors are tested. If this is not the case: actively offered psychosocial care, but not mandatory

8. Results given personally, at the cardiogenetics outpatient clinic, by telephone or by mail, depending on the preference of the individual

9. Actively offered follow-up appointments (including psychosocial care) for mutation carriers, especially those having children

10. Cardiological evaluation and follow-up in mutation carriers, or referral for cardiological evaluation and follow-up to a cardiologist familiar with the disease in the neighbourhood of the residence of the mutation carrier

Adapted from the Huntington guidelines and used by the authors since 1996.

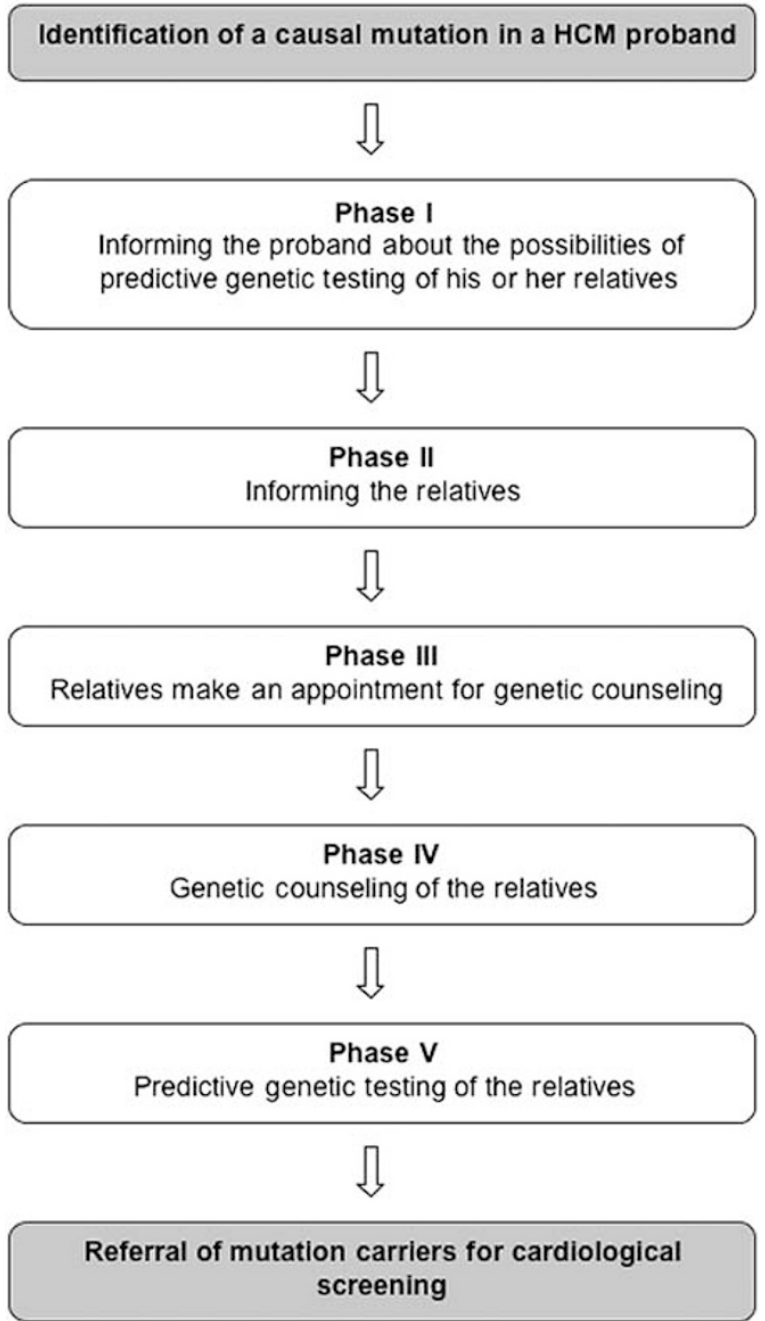

Figure 1 From the detection of a causal mutation in the proband towards cardiological evaluation of mutation carrying relatives.
A proband can do this himself, either orally or by means of a family letter written by the clinical geneticist. This family letter can also be sent directly to all the eligible relatives by the clinical geneticist or relatives can be directly invited for genetic counselling by the clinical geneticist. Probands can be advised in ways to contact their relatives and can be guided which relatives to contact first. In the third phase (III) informed relatives should schedule an appointment for genetic counselling. The fourth phase (IV) is the genetic counselling of the relative. In this phase the pros and cons of the fifth phase $(\mathrm{V})$, predictive DNA testing, are discussed. Carriers of the familial mutation are referred for regular cardiological screening.

\section{Study population}

For this study we included all HCM families $(n=97)$ with a pathogenic mutation of the Academic Medical Centre, Amsterdam identified between 1996, when molecular diagnostics became available in our hospital, and November 2005. A relative was defined eligible for this study when predictive DNA testing was offered. Qualifying for predictive DNA testing in our centre are first-degree relatives, and second-degree relatives in case of a deceased firstdegree relative, of 10 years of age or older.

\section{Analysis}

Data were collected through retrospective audit of pedigrees and medical records and databases. The number of relatives that came for genetic counselling and/or predictive DNA testing in the first year after the detection of the mutation in the proband and their degree of kinship with the proband were assessed. In the Netherlands DNA testing of relatives is always performed in the DNA laboratory where the proband was tested even when genetic counselling took place in another cardiogenetics outpatient clinic, and mutation carriers are lodged into a 
national database (www.gencor.nl), which makes tracking of all relatives counselled and tested possible. ${ }^{21}$

Uptake of genetic counselling was defined as 'the number of eligible relatives who attended (phase IV) in the first year after the detection of the mutation relative to the number of all eligible relatives'. Uptake of predictive DNA testing was defined as 'the number of eligible relatives who were genetically tested (phase V) in the first year after the detection of the mutation relative to the number of all eligible relatives'. The conditional uptake of predictive DNA testing was defined as 'the number of eligible relatives who were genetically tested in the first year after the detection of the mutation (phase $\mathrm{V}$ ) relative to the number of eligible relatives who attended for genetic counselling (phase IV)'. In the result section we report the different kinds of uptake as a mean of the uptake in all families.

Variables collected of the proband were gender, mutated gene and occurrence of SCD in the family, defined as SCD in at least one first-degree relative of the proband under the age of 40 years. Variables recorded in relatives were carrier status, age, gender and degree of kinship. Data were statistically analysed with SPSS (version 14.0) statistical software. Student's $t$-test was used for the comparison of normally distributed continuous variables, non-parametric methods for not-normally distributed continuous variables and Pearson's $\chi^{2}$-test for comparisons between dichotomous variables. A $P$-value $<0.05$ (two-sided) was considered statistically significant.

\section{Results}

\section{Study population}

In the 97 families with a pathogenic mutation 84 families have a mutation in the MYBPC3 gene (of which the c.2373_2374insG Dutch founder mutation in 55 families), 9 families have a myosin heavy chain 7 mutation, 2 families have a Troponin T2 mutation and a mutation in the protein kinase AMP-activated noncatalytic Gamma2 gene was present in 2 families. Families were white Caucasian, except for one family of Indian descent. The index patient was male in 57 (59\%) families. The total number of eligible relatives for genetic counselling and predictive genetic testing was 621 (mean (median) 6.40 (5.00) per family), consisting of 507 first-degree relatives and 114 second-degree relatives.

\section{Uptake of genetic counselling}

In total 235 (mean (median) 2.42 (2.00) per family) eligible relatives attended for genetic counselling in the first year. Hence, the uptake of genetic counselling in relatives was $39.0 \%$. In first-degree relatives uptake was $40.4 \%$ and in the second-degree relatives, who were eligible if the connecting first-degree relative had died, uptake of genetic counselling was $27.5 \%(P=0.047)$. The uptake of genetic counselling did not differ by the relative's gender $(38.7 \%$ in male relatives and $39.6 \%$ in female relatives, $P=0.97$ ) nor did the uptake differ significantly between families with or without SCD (49.2\% in SCD positive families and $35.3 \%$ in SCD negative families, $P=0.14$ ). The number of eligible relatives was not different in families with and without SCD. The proband's gender did not influence uptake of genetic counselling as well (mean uptake of genetic counselling $38.2 \%$ in male probands and $40.0 \%$ in female probands; $P=0.80)$. Uptake of genetic counselling in relatives aged $10-18$ years was $56.1 \%$ (in 22 of 97 families) and in adult relatives uptake was $37.2 \%(P=0.090)$. In 29 (30\%) families no relative attended the outpatient clinic for genetic counselling in the first year. In 16 families relatives attended after this year, but from 13 families (13\%) the relatives have never visited our outpatient clinic; mean follow-up was 53 months (range 16-103).

\section{Uptake of predictive genetic testing}

Of the 235 relatives who attended counselling 233 also proceeded with predictive DNA testing. Two first-degree relatives did not volunteer for predictive DNA testing for reasons of life insurance application. Hence uptake of predictive DNA testing in first-degree relatives was $38.6 \%$. The conditional uptake of predictive DNA testing was $99 \%$ (233 of 235).

\section{Discussion}

Our study, that is the first to systematically evaluate uptake in a cardiogenetic disease, shows that less than half of the eligible relatives from HCM families attended for genetic counselling (phase IV) in the first year after the detection of a pathogenic mutation in the proband. Conditional uptake of predictive DNA testing however was high (phase V). Neither the proband's nor the relative's gender did influence uptake of genetic counselling. A positive family history for SCD and young age ( $<18$ years) did not influence uptake significantly. A larger study must be conducted to show whether uptake of genetic counselling is higher in families with SCD or in young relatives, as the small numbers in our study suggest.

\section{Uptake in other diseases}

Our findings are comparable to uptake of genetic counselling in other genetic late-onset diseases that have preventive options, like hereditary breast cancer and hereditary non-polyposis colorectal cancer, in which uptake varies between 23 and $45 \% .^{22-26}$ With an active approach (directly contacting all relatives by telephone or mail) for research purposes uptake rates of $51 \%$ and even $78 \%$ have been reported. ${ }^{22,27}$ Uptake of counselling in relatives from families with Duchenne or Becker muscular dystrophy, myotonic dystrophy or a balanced chromosome translocation also reveals similar uptake percentages $(30 \%) .^{28}$ 
Only Charron et $a l^{29}$ provide information on the results of counselling and genetic testing for HCM in 70 subjects (29 adults attending for predictive testing, 9 couples requesting predictive testing for their children, 22 couples consulting for prenatal counselling and 10 probands who came for diagnostic testing). A counselling session consisted of consecutive interviews with a cardiologist, a clinical geneticist and a psychologist in the same place and during the same visit. There was a delay before the decision about genetic DNA testing, following the Huntington guidelines. In total 10 out of 29 adults chose to decline predictive genetic testing after one genetic counselling session. Conditional uptake of genetic DNA testing was not reported as so, but was $45 \%$ when calculated. After the announcement of the result, follow-up consisted of regular interviews and psychosocial support if necessary. No adverse effects were reported during a mean follow-up of 8 months, except for one woman with subdepressive syndrome.

\section{What determines uptake?}

As the conditional uptake of predictive DNA testing in our study is almost $100 \%$ (phase IV/V in Figure 1), the reasons for suboptimal uptake in our HCM families must therefore relate to phase I, II or III. In phase I the proband must be informed about the possibility of predictive DNA testing in relatives. As genetic counsellors we must ascertain that the proband fully understands the information received. In phase II, the information from phase I should be disseminated to the proband's relatives. Differences in uptake in phase II may originate from at least three sources: which information is disseminated, how the information is transferred and by whom. The counsellor can also be significant in safeguarding this process. In our setting the probands were informed about the possibilities of predictive DNA testing in relatives by a genetic counsellor. Eligible relatives were identified in the pedigree and a family letter was provided to help inform the relatives. If necessary the proband could get help from a social worker to give social support and strategies in informing his or her relatives. Informing the relatives (phase II) can also be performed in a more active way by the genetic counsellor, but this was not our common practice in the families in our study. Studies that use active recruitment as part of their research protocol generally report higher uptake rates. ${ }^{22,24,25,27}$ The study of Suthers $e^{2} a^{26}$ demonstrates the rise in uptake of genetic testing for familial cancer syndromes (from 13 to $40 \%$ ) when relatives are informed by a letter directly sent to them by the clinical geneticist. They report that $7 \%$ of relatives did not want further information after receiving the letter and none of the 351 relatives complained of a breach of privacy or autonomy. ${ }^{26}$ In the Netherlands cascade screening for familial hypercholesterolemia with a direct active approach in contacting the relatives is well accepted..$^{30}$ Interviews of probands and relatives referred for genetic counselling for Duchenne or Becker muscular dystrophy, myotonic dystrophy or a balanced chromosome translocation showed that $98 \%$ of probands and $94 \%$ of relatives thought it was acceptable for relatives to be informed of their risk status by another family member, and $78 \%$ of probands and $90 \%$ of relatives had no objection to this information coming directly from the clinical genetic service. ${ }^{28,31}$ Factors influencing uptake in phase III, relatives making an appointment for genetic counselling, can be found in a model of health care developed by Andersen. According to Andersen's model, suboptimal uptake may relate to either of the following three characteristics influencing the use of health services: need variables (variables influencing the need for care, eg health education programmes or a family letter from the clinical geneticist), predisposing variables (age, gender, social structure, health beliefs, perceptions) and enabling variables (community and personal enabling resources, eg income, health insurance or travel time). ${ }^{32}$ We could not demonstrate that uptake was influenced by variables like age, gender or a family history of SCD. Degree of kinship did influence uptake in our study.

The uptake of phase IV, genetic counselling of the relatives, mainly depends on how successful the first two phases were and on the personal motives or life events that may affect a relative's decision to enter phase III. We retrospectively measured uptake within the first year after the mutation was detected in the proband, to be able to compare families and to give relatives some time for reflection. This time horizon, however, may exclude relatives with personal motives or life events preventing them to attend within the first year.

In the counselling session (phase IV) the relative is informed about the advantages and disadvantages of DNA testing. This can be performed in one session or in several sessions with time for reflection. Uptake of predictive genetic testing (phase V) may be influenced by the information given by the clinical geneticist, treatment options for the disease and time given for reflection. Unlike in literature on uptake in oncogenetics, in other late onset hereditary diseases, and in the relatives from HCM families described by Charron et $a l^{29}$, in our outpatient clinic almost all relatives who attend for genetic counselling for $\mathrm{HCM}$ proceeded in predictive genetic testing. ${ }^{33,34}$ In most studies reported in literature the counsellors used several counselling sessions, according to the Huntington guidelines. In contrast to these guidelines, we chose for several reasons to reduce the time for reflection before predictive DNA testing. This might not give relatives sufficient time to think over all consequences and undeliberately coerce them to get tested. Brain et $a l^{35}$, however, demonstrated that a shortened counselling process in oncogenetics without a reflection period did not cause psychological harm and did not affect the decision-making process of undergoing DNA testing. Currently the counselling process 
experienced by the counselees from HCM families in our centre is being evaluated.

For late-onset disorders generally the prognosis and the opportunities of preventive measures determine the rate of uptake of DNA testing. ${ }^{23}$ The age of disease onset might also be a determinant of the uptake rate. Because HCM is a disease that can already become manifest in adolescence and is associated with unexpected SCD that can be prevented, uptake of DNA testing can theoretically expected to be higher than in other preventable late-onset disorders.

\section{Should uptake be increased?}

From a public health point of view uptake of predictive DNA testing (phase V) in HCM should be as high as possible to prevent SCD in identified high-risk individuals. ${ }^{18}$ From a clinical genetic point of view we also emphasize to inform all relatives about the possibility of predictive testing, but proceeding with genetic testing should be their own well-informed choice. Uptake of genetic counselling (phase IV) from both viewpoints should ideally be $100 \%$. However, after being wellinformed it is to be expected that not all relatives will proceed with predictive testing.

In our outpatient clinics we can ascertain that the proband fully understands the possibilities of DNA testing of his or her relatives (phase I). Contacting the proband's relatives (phase II) either through the proband or actively by the clinical genetic service raises ethical and legal issues. In particular, there could be an ethical tension between maximizing the utility of cascade screening and respecting the interests of probands and their relatives. Relatives are said to have 'the right not to know' and clinicians have an obligation to protect the confidentiality of the proband, but this must be balanced with the central purpose of cascade testing; informing relatives and promoting autonomous decision-making by relatives about their genetic risk. This purpose is counteracted by inefficient or ineffective family communication processes. ${ }^{36}$ It is also important to recognize that the legal applicability of the two contact strategies may vary between countries depending on legal rules and a country's system of health care. ${ }^{37}$ In our HCM families, relatives were informed by means of a family letter and uptake of genetic counselling was not optimal, but conditional uptake of predictive DNA testing was almost $100 \%$. This could imply that offering relatives counselling (phase II), for example by means of a family letter in which the disease and options for predictive genetic testing and prevention are already explained, is some sort of genetic counselling in itself.

To increase uptake of genetic counselling, determinants that can be influenced should be looked for in phase I, II and III. Further research into the determinants of uptake with a prospective design is needed. A comparison with other late-onset hereditary diseases, in which uptake has been studied more thoroughly, could provide more information as well.

\section{Acknowledgements}

Clinical data of the HCM families in this study are lodged in the GENCOR database (www.gencor.nl), a national database for familial heart diseases, supported by the Interuniversity Cardiology Institute of the Netherlands. This research is financially supported by ZorgOnderzoek Nederland (ZonMw), grant number 62000010. The funding organisation has had no involvement in study design, collection, analysis and interpretation of data; in the writing of this paper and in the decision to submit the paper for publication. There are no actual or potential conflicts of interest for all authors.

\section{References}

1 Maron BJ, Gardin JM, Flack JM, Gidding SS, Kurosaki TT, Bild DE: Prevalence of hypertrophic cardiomyopathy in a general population of young adults. Echocardiographic analysis of 4111 subjects in the CARDIA Study. Coronary artery risk development in (young) adults. Circulation 1995; 92: 785-789.

2 Morita H, Larson MG, Barr SC et al: Single-gene mutations and increased left ventricular wall thickness in the community: the Framingham Heart Study. Circulation 2006; 113: 2697-2705.

3 Zou Y, Song L, Wang Z et al: Prevalence of idiopathic hypertrophic cardiomyopathy in China: a population-based echocardiographic analysis of 8080 adults. Am J Med 2004; 116: 14-18.

4 Maron BJ: Hypertrophic cardiomyopathy: a systematic review. JAMA 2002; 287: 1308-1320.

5 Cannan CR, Reeder GS, Bailey KR, Melton III LJ, Gersh BJ: Natural history of hypertrophic cardiomyopathy. A population-based study, 1976 through 1990. Circulation 1995; 92: 2488-2495.

6 Maron BJ, Olivotto I, Spirito P et al: Epidemiology of hypertrophic cardiomyopathy-related death: revisited in a large non-referralbased patient population. Circulation 2000; 102: 858-864.

7 de Vreede-Swagemakers JJ, Gorgels AP, Dubois-Arbouw WI et al: Out-of-hospital cardiac arrest in the 1990's: a population-based study in the Maastricht area on incidence, characteristics and survival. J Am Coll Cardiol 1997; 30: 1500-1505.

8 Girolami F, Olivotto I, Passerini I et al: A molecular screening strategy based on beta-myosin heavy chain, cardiac myosin binding protein $\mathrm{C}$ and troponin $\mathrm{T}$ genes in Italian patients with hypertrophic cardiomyopathy. I Cardiovasc Med (Hagerstown) 2006; 7: 601-607.

9 Richard P, Charron P, Carrier L et al: Hypertrophic cardiomyopathy: distribution of disease genes, spectrum of mutations, and implications for a molecular diagnosis strategy. Circulation 2003; 107: 2227-2232.

10 Alders M, Jongbloed R, Deelen W et al: The 2373insG mutation in the MYBPC3 gene is a founder mutation, which accounts for nearly one-fourth of the HCM cases in the Netherlands. Eur Heart J 2003; 24: 1848-1853.

11 Elliott PM, Poloniecki J, Dickie S et al: Sudden death in hypertrophic cardiomyopathy: identification of high risk patients. J Am Coll Cardiol 2000; 36: 2212-2218.

12 Elliott PM, Gimeno JR, Tome MT et al: Left ventricular outflow tract obstruction and sudden death risk in patients with hypertrophic cardiomyopathy. Eur Heart J 2006; 27: 1933-1941.

13 Kofflard MJ, Ten Cate FJ, van der Lee C, van Domburg RT: Hypertrophic cardiomyopathy in a large community-based population: clinical outcome and identification of risk factors for sudden cardiac death and clinical deterioration. J Am Coll Cardiol 2003; 41: 987-993.

14 Niimura H, Bachinski LL, Sangwatanaroj S et al: Mutations in the gene for cardiac myosin-binding protein $\mathrm{C}$ and late-onset familial hypertrophic cardiomyopathy. $N$ Engl J Med 1998; 338: $1248-1257$. 
15 Maron BJ, McKenna WJ, Danielson GK et al: American College of Cardiology/European Society of Cardiology clinical expert consensus document on hypertrophic cardiomyopathy. A report of the American College of Cardiology Foundation Task Force on Clinical Expert Consensus Documents and the European Society of Cardiology Committee for Practice Guidelines. I Am Coll Cardiol 2003; 42: 1687-1713.

16 Maron BJ, Estes III NA, Maron MS, Almquist AK, Link MS, Udelson JE: Primary prevention of sudden death as a novel treatment strategy in hypertrophic cardiomyopathy. Circulation 2003; 107: 2872-2875.

17 Maron BJ, Spirito P, Shen WK et al: Implantable cardioverterdefibrillators and prevention of sudden cardiac death in hypertrophic cardiomyopathy. JAMA 2007; 298: 405-412.

18 Marteau TM, Kinmonth AL: Screening for cardiovascular risk: public health imperative or matter for individual informed choice? BMJ 2002; 325: 78-80.

19 van Langen I, Hofman N, Tan HL, Wilde AA: Family and population strategies for screening and counselling of inherited cardiac arrhythmias. Ann Med 2004; 36 (Suppl 1): 116-124.

20 ASHG statement: Professional disclosure of familial genetic information. The American Society of Human Genetics Social Issues Subcommittee on Familial Disclosure. Am J Hum Genet 1998; 62: 474-483.

21 Hermans J, Christiaans I, van Tintelen J, Wilde A, Pinto Y: GENCOR: a national registry for patients and families suffering from a familial heart disease in the Netherlands. Neth Heart J 2006; 14: 274-278.

22 Aktan-Collan K, Mecklin JP, Jarvinen $\mathrm{H}$ et al: Predictive genetic testing for hereditary non-polyposis colorectal cancer: uptake and long-term satisfaction. Int J Cancer 2000; 89: 44-50.

23 Brooks L, Lennard F, Shenton A et al: BRCA1/2 predictive testing: a study of uptake in two centres. Eur J Hum Genet 2004; 12: 654-662.

24 Codori AM, Petersen GM, Miglioretti DL et al: Attitudes toward colon cancer gene testing: factors predicting test uptake. Cancer Epidemiol Biomarkers Prev 1999; 8: 345-351.

25 Lerman C, Hughes C, Trock BJ et al: Genetic testing in families with hereditary nonpolyposis colon cancer. JAMA 1999; 281: $1618-1622$.

26 Suthers GK, Armstrong J, McCormack J, Trott D: Letting the family know: balancing ethics and effectiveness when notifying relatives about genetic testing for a familial disorder. I Med Genet 2006; 43: 665-670.

27 Hadley DW, Jenkins J, Dimond E et al: Genetic counseling and testing in families with hereditary nonpolyposis colorectal cancer. Arch Intern Med 2003; 163: 573-582.

28 Kerzin-Storrar L, Wright C, Williamson PR et al: Comparison of genetic services with and without genetic registers: access and attitudes to genetic counselling services among relatives of genetic clinic patients. J Med Genet 2002; 39: 85e.

29 Charron P, Heron D, Gargiulo M et al: Genetic testing and genetic counselling in hypertrophic cardiomyopathy: the French experience. J Med Genet 2002; 39: 741-746.

30 van Maarle MC, Stouthard ME, Marang-van de Mheen PJ, Klazinga NS, Bonsel GJ: How disturbing is it to be approached for a genetic cascade screening programme for familial hypercholesterolaemia? Psychological impact and screenees' views. Community Genet 2001; 4: 244-252.

31 Wright C, Kerzin-Storrar L, Williamson PR et al: Comparison of genetic services with and without genetic registers: knowledge, adjustment, and attitudes about genetic counselling among probands referred to three genetic clinics. J Med Genet 2002; 39: $84 \mathrm{e}$.

32 Andersen RM: Revisiting the behavioral model and access to medical care: does it matter? J Health Soc Behav 1995; 36: $1-10$.

33 Biesecker $\mathrm{BB}$, Ishibe N, Hadley DW et al: Psychosocial factors predicting BRCA1/BRCA2 testing decisions in members of hereditary breast and ovarian cancer families. Am J Med Genet 2000; 93: 257-263.

34 Foster C, Evans DG, Eeles R et al: Predictive testing for BRCA1/2: attributes, risk perception and management in a multi-centre clinical cohort. Br J Cancer 2002; 86: 1209-1216.

35 Brain K, Sivell S, Bennert K et al: An exploratory comparison of genetic counselling protocols for HNPCC predictive testing. Clin Genet 2005; 68: 255-261.

36 Gaff CL, Clarke AJ, Atkinson P et al: Process and outcome in communication of genetic information within families: a systematic review. Eur J Hum Genet 2007; 15: 999-1011.

37 Newson AJ, Humphries SE: Cascade testing in familial hypercholesterolaemia: how should family members be contacted? Eur J Hum Genet 2005; 13: 401-408. 